\title{
Concurso Público. Rehabilitación y restauración Casa Central de la Universidad de Chile
}

NOMBRE DE LA OBRA: Rehabilitación y Restauración Casa Central de la Universidad de Chile - MANDANTE: Universidad de Chile - ARQUITECTOS RESPONSABLES: Primer Premio: Rodrigo Chauriye Ch + Luis Alberto Reyes S + Álvaro Farrú B. / Arquitectos Asociados: Beatriz Stäger R + Osvaldo Moreno F • SUPERFICIE TERRENo: $5.850 \mathrm{M}^{2} \bullet$ SUPERFICIE CONSTRUIDA: $6.565 \mathrm{M}^{2} \bullet$ ANNO PROYECTO: 2008 - MATERIALES PREDOMINANTES: Hormigón armado + Madera + Vidrio

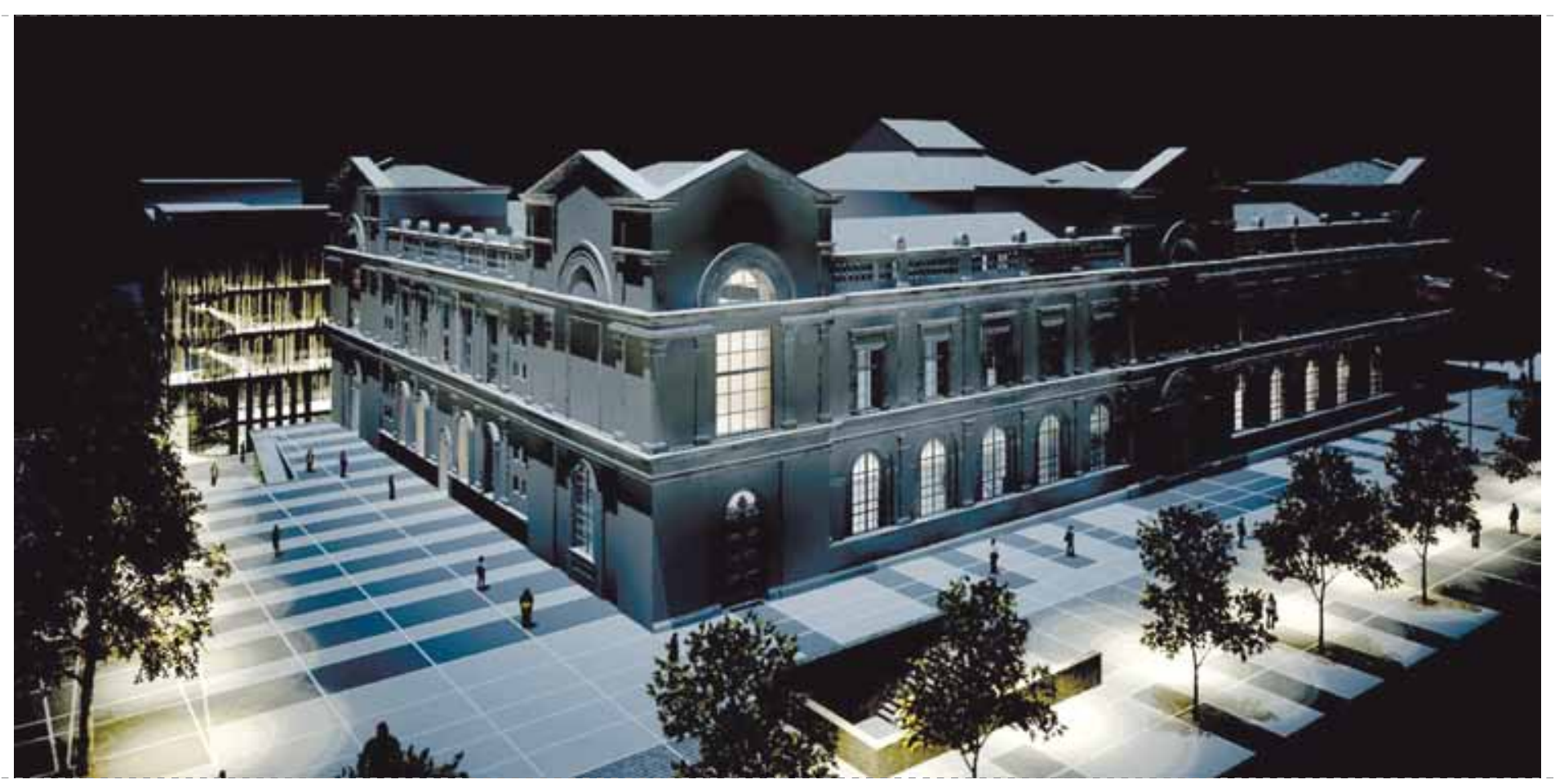

Tradición y modernidad

La Universidad de Chile se erigió desde sus inicios en ente rector y pieza fundacional de la educación superior chilena y, bajo esta óptica, su Casa Central representa los ideales intelectuales, científicos y artísticos que han forjado la vida republicana de Chile desde hace casi dos siglos.

Desde esta perspectiva, nuestra propuesta plantea recuperar este sitial histórico a través de la revalorización de la arquitectura del edificio primario de la universidad y recuperar la presencia y solemnidad de sus espacios emblemáticos. Para esto, se decide limpiar los espacios del primer piso, o piso noble, eliminando la constricción y subdivisión actual de éstos en pequeños y pobres recintos, devolviéndoles su escala, altura y proporciones originales, para así otorgarles la severidad y la nobleza que el edificio histórico planteó en su origen.
Esto se refuerza mediante la asunción por parte de estos espacios de las funciones fundamentales de la universidad, aquellas que la han distinguido a nivel nacional e internacional, tales como extensión cultural (librería, biblioteca, exposiciones artísticas), carácter público (plaza abierta a la comunidad) y jerarquía institucional (rectoría). En este hecho descrito se pretende reforzar la vocación pública de la Universidad de Chile y su aporte al país, rescatando la condición actual de la institución en su doble cualidad, tanto como 
símbolo de tradición intelectual y científica de Chile, como asimismo la vanguardia de una universidad que se proyecta hacia el siglo xxI asumiendo nuevos y variados desafíos.

\section{Memoria histórica y vanguardia científica de la Universidad de Chile}

En esta línea, y con el ánimo de reordenar funciones y limpiar los espacios actuales, se ha determinado como uno de los ejes fundamentales de la propuesta, la creación de un volumen que permita reutilizar de mejor forma el deslinde sur del predio y reubicar variadas funciones de la universidad, tanto nuevas como tradicionales, que generan en la actualidad un fuerte deterioro del edificio histórico o están relegadas a un segundo plano a pesar de su importancia.

Este nuevo volumen se consolida como un bloque espacial y funcional, que limita y a la vez integra la Casa Central con el Instituto Nacional, restituyendo su relación histórica a través de la inserción en él de un programa eminentemente intelectual y científico, como son el Archivo Central de la Universidad de Chile y el área de investigación.

Estos dos polos de desarrollo universitario contenidos en el nuevo edificio constituyen tanto la memoria histórica como la vanguardia científica del país, vistas y analizadas a través de los ojos de la Universidad de Chile, hechos que sintetizan tradición y modernidad, así como pasado y presente, constituyéndose en los motores de desarrollo más importantes de la universidad. Por lo mismo, proponemos que, como nombre tentativo y a modo de homenaje, este nuevo bloque de la memoria podría denominarse edificio Miguel Luis Amunátegui, en honor al gran historiador, político y académico de la universidad y profesor del Instituto Nacional.

El nuevo volumen se separa de la Casa Central a través de una materialidad translúcida y etérea que contrasta con la opacidad y solidez del actual edificio, y funcional y espacialmente lo hace a través de un espacio longitudinal de circulación de toda su altura, que contiene en su fondo un espejo de agua, vegetación acuática y la inserción en él de esculturas desarrolladas por artistas formados en la universidad. El volumen propuesto desea destacarse no sólo por su carácter funcional, sino especialmente por esa doble condición, tan propia de la actividad universitaria: transparencia y dinamismo, símbolos de una Universidad de Chile que se abre al siglo xxı,
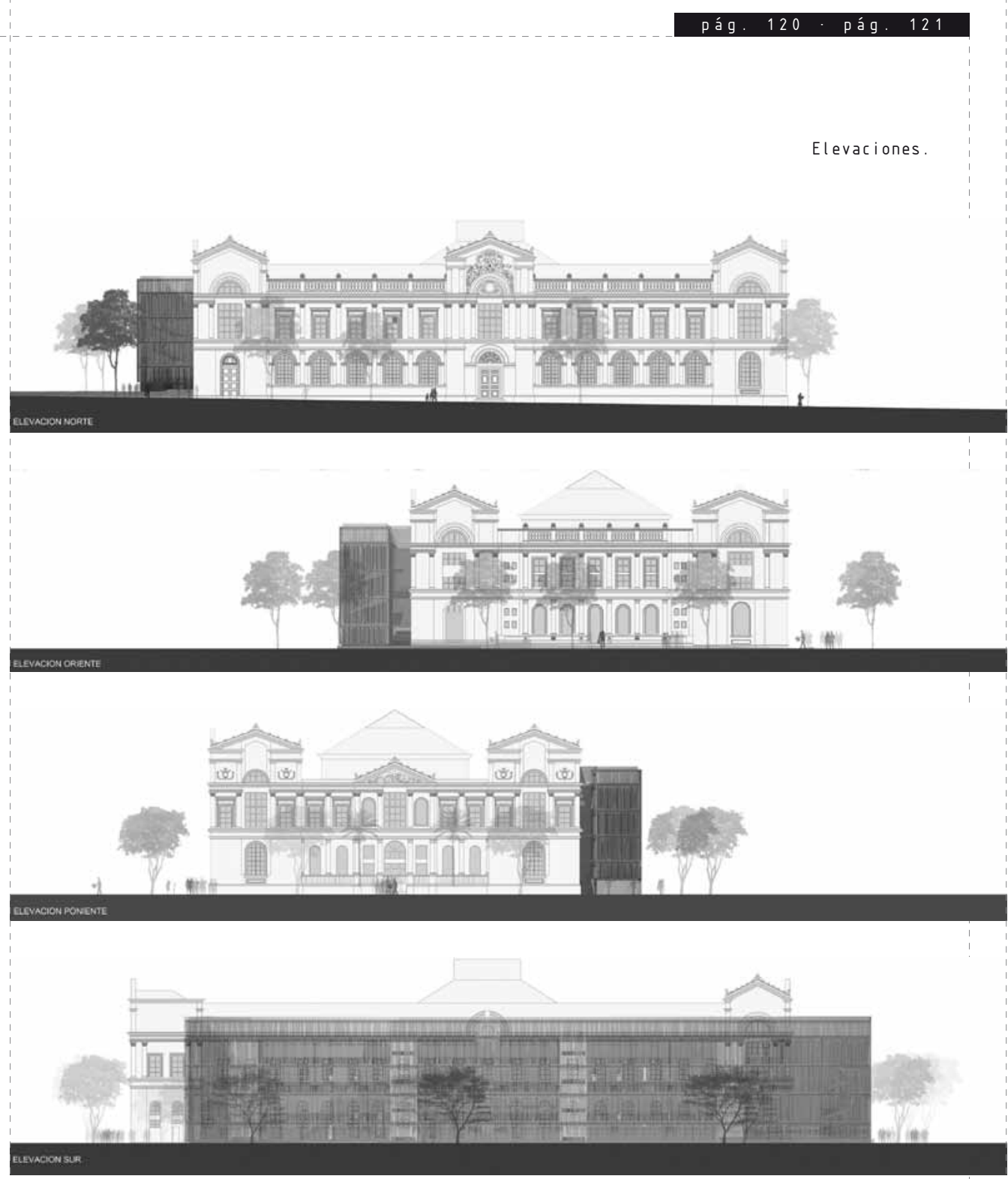

cualidades que están determinadas por su materialidad exterior y su estructura interna.

\section{Plaza de los Presidentes}

El otro eje principal de la propuesta es la creación de una plaza en el costado oriente del edificio actual, la cual se constituye como una prolongación de la zona peatonal de calle Arturo Prat hacia el poniente, unificándose en un solo gran espacio público, que permite acceder a ciertas actividades de la universidad y a su vez le da un marco adecuado al edificio histórico, permitiendo la visión y el goce de la totalidad de su arquitectura.

Esta plaza, la cual hemos denominado Plaza de los Presidentes debido a la gran cantidad de ex presidentes de nuestro país que han pasado por las aulas de la Universidad de
Chile, contiene bajo ella el teatro para música de cámara y cine con capacidad para 400 espectadores, la nueva sala Ignacio Domeyko, a la cual se puede acceder desde la misma plaza y a través de un foyer ubicado en el nuevo edificio.

Finalmente, se ha optado por desplazar el monumento de Miguel Luis Amunátegui desde su posición actual hacia la Alameda, de tal forma de generar una plaza ininterrumpida, alinear el monumento con el acceso a la estación del metro y con el monumento a Andrés Bello y crear un gran espacio público que unifique la calzada de la universidad frente a la Alameda Bernardo O'Higgins, dando forma al nuevo atrio urbano: el espacio ceremonial que el edificio de la Casa Central merece. 


\section{Sala Ignacio Domeyko}

la ubicación de una pieza de la envergadura del teatro bajo la plaza, tiene la finalidad de potenciarla como espacio público de gran vitalidad y, sobre todo, de evitar sobrecargar el edificio histórico con espacios que requieren soluciones funcionales y estructurales complejas, lo que supondría un problema más que una solución.

De este modo, la sala se comunica con otros recintos de similares características, creando un ámbito cultural que comunica el espacio bajo la plaza con el piso zócalo del nuevo edificio y con el subsuelo del patio oriente del edificio histórico.

\section{Criterios de restauración}

Uno de los elementos centrales es plantear la intervención global como una propuesta de restauración integral del edificio histórico, tanto desde el punto de vista estructural como de formal y, especialmente, desde el funcional, pero sin alterar el carácter y la fisonomía originales del edificio.

\section{Limpieza espacial}

Por lo mismo, una de las primeras y más importantes decisiones es despejar y limpiar el interior del edificio en sus pisos nobles para devolverle a éstos su carácter y prestancia originales, lo que se refuerza cambiando el pavimento en los patios y hall de ingreso y modificando el color existente en la fachada, pintando tanto exterior como interiormente el edificio de blanco, simbolizando de esta manera el carácter esencial y severo de la Universidad de Chile.

\section{Refuerzos estructurales}

Otro eje de la restauración la constituyen los refuerzos estructurales que se aplicarán a las zonas más dañadas, especialmente los dinteles y columnas de los patios, ya que la propuesta plantea cambiar la cubierta de ambos patios, lo que puede provocar variaciones de peso respecto a las actuales, las que ya hoy en día sobrecargan la estructura de los dinteles, especialmente en las esquinas.

En esta misma lógica, se reforzarán con estructuras de acero las cadenas y vigas que reciben los entramados de piso, los cuales, dependiendo de su estado, se cambiarán por losas colaborantes de acero y hormigón armado para evitar sobrecargar los muros del edificio más allá de lo aceptable. A esto se
Vista aérea general
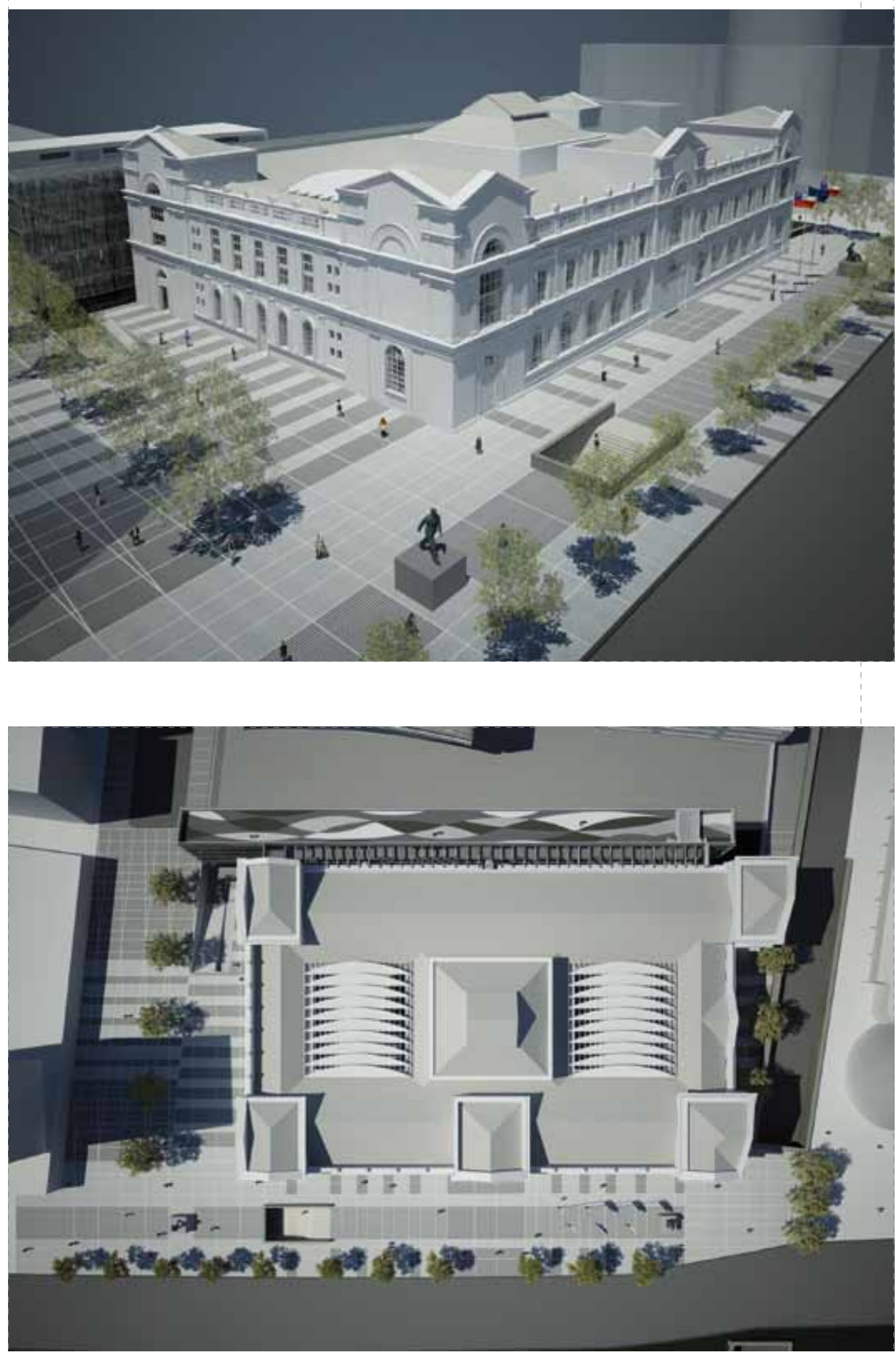

Planta definitiva. 
Sala

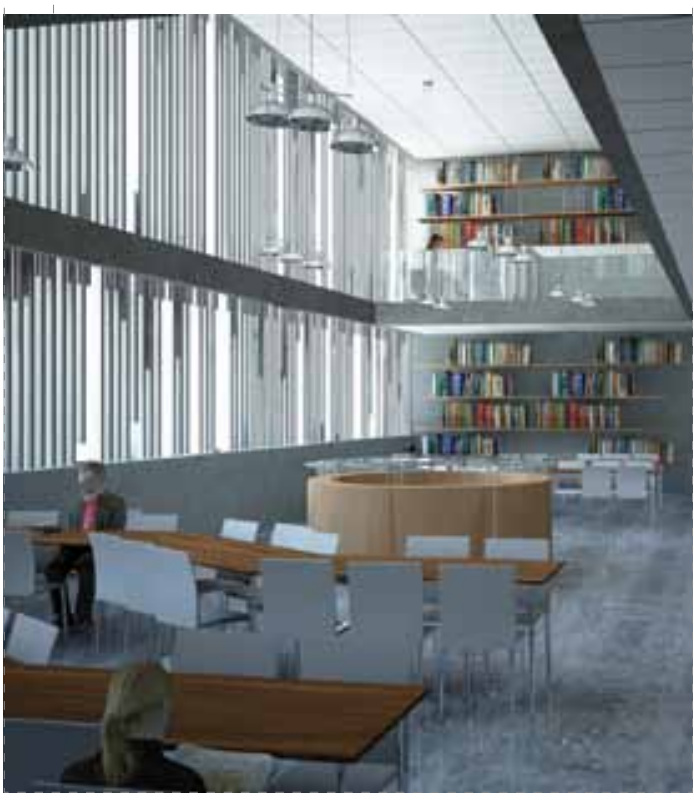

sumará el cambio o refuerzo de las losas de los patios debido al aumento de la superficie edificada bajo ellos.

Como parte de esta restauración estructural, y aprovechando la decisión de modificar las cubiertas, se ha optado por reemplazar las actuales cerchas de madera por cerchas metálicas que permitan sustentar la nueva configuración de las cubiertas y la aplicación de aislamiento.

\section{Valorización ornamental}

Más allá de los temas estructurales de edificio histórico, la propuesta se aleja de pintoresquismos, caricaturizaciones o restauraciones críticas, conceptos que no se condicen con el estado actual del edificio, con los conceptos de tradición y modernidad unidos en un solo ámbito, ni mucho menos con el hecho de que los espacios seguirán absorbiendo funciones dinámicas que suponen más que nada una restauración de aquellos sectores altamente deteriorados $y$, sobre todo, de un trabajo que destaque y ponga en valor los principios neoclásicos del inmueble, tales como cornisas, balaustras, artesonados, capiteles, puertas, ventanas y pinturas, entre otros aspectos, recuperando su impronta y lozanía originales.

Desde este punto de vista, una intervención mayor que se propone para restaurar la grandeza de un espacio emblemático es el hecho de rematar la bóveda del salón de honor con una pintura acorde a su envergadura,
Patio

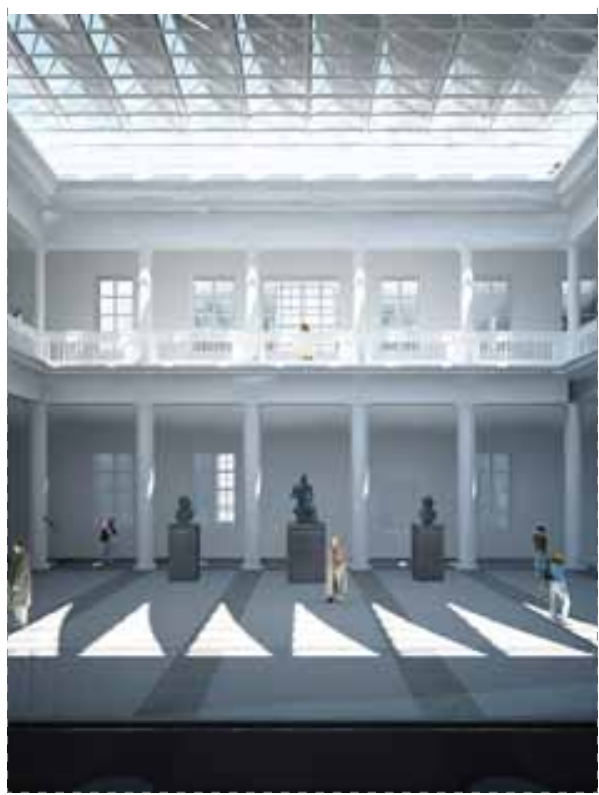

desarrollada por un artista formado en la Universidad de Chile, desde una óptica contemporánea, pero con respeto al pasado y a la arquitectura del edificio.

\section{Eficiencia energética y sustentabilidad}

Dentro de la propuesta se le ha dado gran importancia al tema energético del edificio, algo que una institución como ésta y un edificio de esta envergadura deben sobrellevar equilibradamente, por lo que se ha considerado dotar tanto al edificio histórico como al nuevo volumen de sistemas de captación de energía para procurar una eficiencia energética y sustentabilidad ambiental, conceptos imprescindibles frente a la actual crisis de energía.

\section{Aprovechamiento exposición} solar de techumbres

De esta manera, la solución propuesta plantea reemplazar las actuales cubiertas del edificio histórico por paneles solares, los cuales absorben la energía calórica y las radiaciones ultravioletas del sol, calentando agua, la que se conduce a termos acumuladores de agua caliente ubicados bajo la cubierta, entre cerchas, y de ahí se distribuye al resto de edificio a través de losas y entrepisos.

La gran cantidad de superficie de cubiertas expuestas al norte permite utilizar el sistema descrito sin modificar la fisonomía actual de la

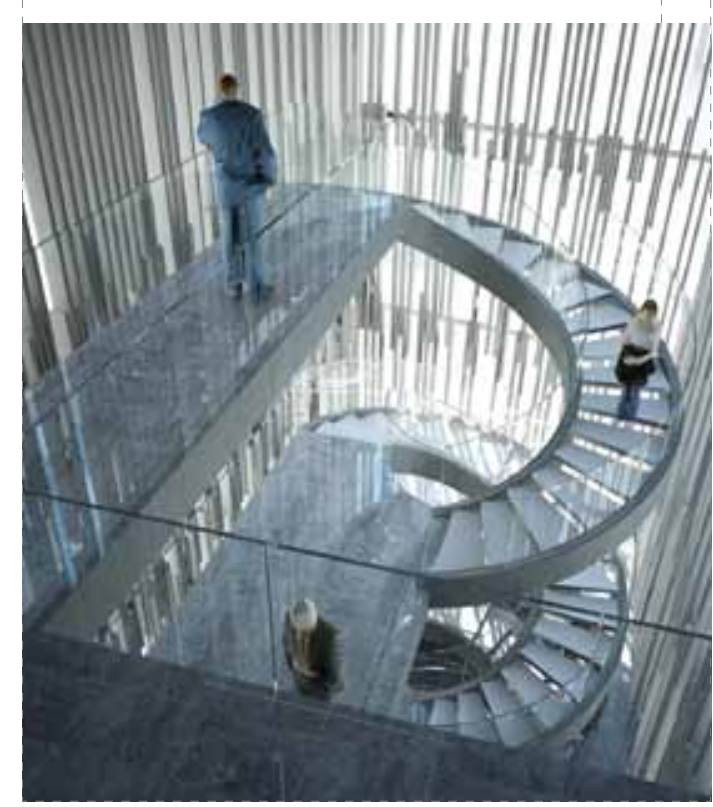

techumbre y paliar en un rango aproximado del $15 \%$ al $25 \%$ el gasto energético de la Casa Central, tanto en calefacción como en agua caliente.

\section{Cubierta fotovoltaica en patios}

En la idea de manejar más y mejores recursos energéticos para el edificio, la propuesta plantea aprovechar una premisa de diseño del proyecto, la cual es modificar las cubiertas de ambos patios, e insertar en ellas paneles fotovoltaicos con placas monocristalinas, que aportarían a la generación de energía eléctrica para los edificios.

Como dato a considerar para definir el planteamiento de las cubiertas de los patios, se tomó en consideración que las placas aportan en promedio en un día soleado alrededor de $120 \mathrm{w} / \mathrm{m}^{2}$, por lo que cada patio, que cuenta con una superficie de cubierta de $250 \mathrm{~m}^{2}$ aportaría alrededor de 30.000 w al sistema de energía eléctrica en condiciones ideales.

Independientemente de la incorporación de los paneles fotovoltaicos, las cubiertas de los patios siguen manteniendo las actuales condiciones de transparencia que permite contar con iluminación solar directa durante el día, dándole con el nuevo diseño un valor agregado en términos de energía y la posibilidad de matizar la luz y el calor directos sobre los patios para crear un espacio lumínico y térmicamente controlado, evitando el deterioro en el tiempo de pavimentos y pinturas. 